\title{
Effect of recombinant TRAIL in a murine co-culture system of osteoclastogenesis
}

\author{
V. Nicolin, P. Narducci, C. Celeghini \\ Department of Biomedicine, University of Trieste, Trieste, Italy
}

(C2008 European Journal of Histochemistry

Although some experimental evidence has implicated the TRAIL/TRAIL-receptor system in the regulation of osteoclastogenesis, the only available studies performed so far have been performed on isolated pre-osteoclasts, induced to differentiate by the addition of recombinant RANKL and M-CSF. Using a more physiological co-culture system in the absence of exogenous cytokines, we have here demonstrated that recombinant TRAIL inhibits osteoclast formation, but only at relatively high concentrations $(500 \mathrm{ng} / \mathrm{mL})$.

Key words: TRAIL, osteoclastogenesis, OPG.

Correspondence: Claudio Celeghini,

Department of Biomedicine, University of Trieste,

Via Manzoni 16, 34138 Trieste, Italy

Tel: +39.040 .5586007 .

Fax: +39.040.5586016

E-mail: cceleghini@units.it.

Paper accepted on February 7, 2008

European Journal of Histochemistry

2008; vol. 52 issue 1 (Jan-Mar): 75-78
A number of studies have suggested a potential role of the tumor necrosis factor (TNF)-related apoptosis inducing ligand (TRAIL) in modulating the differentiation of hematopoietic progenitors towards different lineages, including osteoclastogenesis (Secchiero et al., 2002; Secchiero et al., 2004; Zauli et al., 2004). In particular, previous studies have shown that recombinant TRAIL either inhibits both human and mouse osteoclastogenesis when added to isolated preosteoclasts induced to differentiate with soluble recombinant macrophage colony stimulating factor $(\mathrm{M}-\mathrm{CSF})+$ receptor activator of NF-kB ligand (RANKL) (Zauli et al., 2004; Zauli et al., 2008) or induce apoptosis of mature osteoclasts (Roux et al., 2005, Colucci et al. 2007). On the other hand, another study performed on peripheral blood cells purified from patients affected by multiple myeloma showed that the addition of recombinant TRAIL paradoxically induced osteoclastogenesis (Colucci et al., 2004). The suggested mechanism proposed by Colucci et al. (2004) to explain the pro-osteoclastic activity of TRAIL was that TRAIL likely precluded osteoprotegerin (OPG) produced by the peripheral blood cells of patients affected by multiple myeloma from inhibiting the pro-osteoclastic activity of RANKL. In this respect, it has been recently shown that the affinity of OPG for native TRAIL was comparable to that for RANKL at $37^{\circ} \mathrm{C}$, as determined by plasmon surface resonance analysis (Vitovski et al., 2007). Moreover, in vitro studies have convincingly demonstrated that $O P G$ acts in a paracrine or autocrine manner by binding TRAIL and promoting the survival of prostate cancer cells (Holen et al., 2002) and multiple myeloma cells (Shipman and Croucher, 2003). Thus, the exact role of TRAIL in modulating osteoclastogenesis is uncertain.

In our opinion, the major limitation of these previous studies is that the effect of TRAIL was evaluated in culture systems in which isolated human peripheral blood mononuclear cells or the 
A

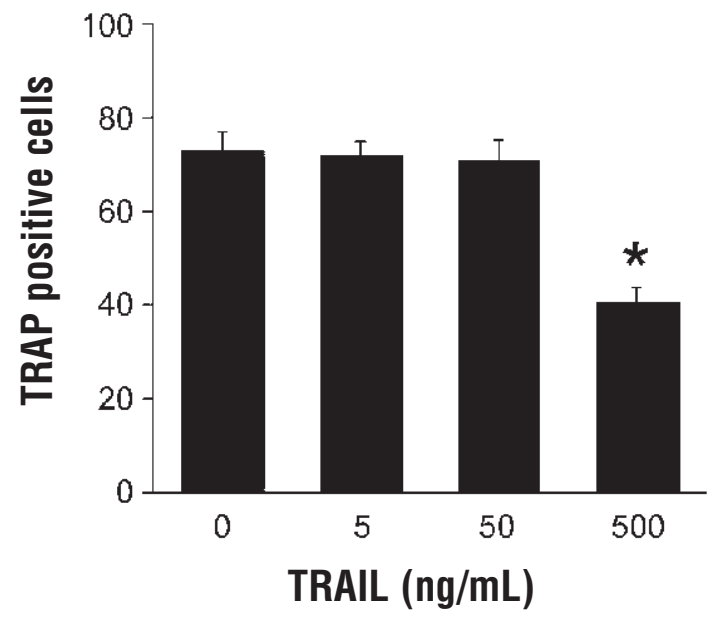

B
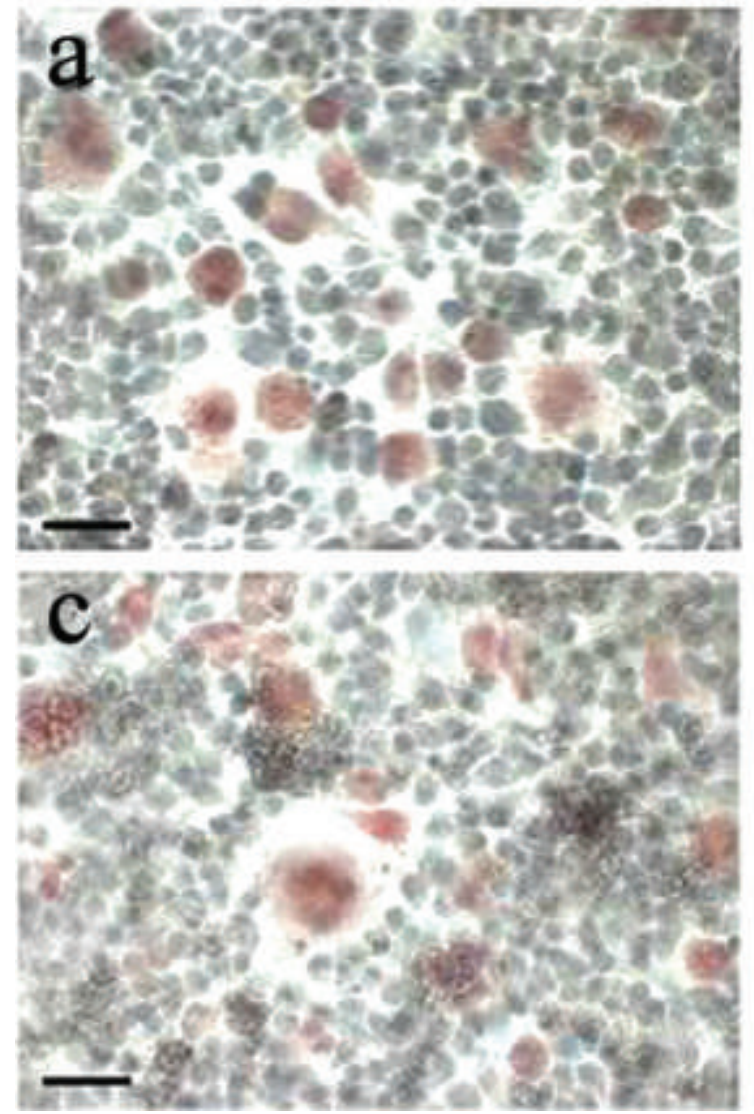

RAW264.7 macrophagic cell line were induced to differentiate into mature osteoclasts by the addition of recombinant RANKL+M-CSF. Since bone marrow stromal cells and osteoblasts are the major source of OPG within the bone marrow environment (Boyle et al., 2003), to shed light into the role of TRAIL in osteoclastogenesis, we have analyzed the

\begin{abstract}
Figure 1. Effect of treatment with increasing concentrations of recombinant TRAIL on osteoclastic differentiation in a RAW264.7/osteoblast co-culture system. After $\mathbf{4}$ days of co-culture of mouse osteoblasts type CRL 12257 with mouse monocytoid RAW264.7 cells, the degree of differentiation was evaluate by scoring the number of the tartrate-resistant acid phosphatase (TRAP)-positive multinucleated cells. In $A$, data are expressed as means + SD of three different experiments performed in duplicate $\left({ }^{*} p<0.05\right)$. In $B$, representative fields of co-cultures treated with vehicle (a), $5 \mathrm{ng} / \mathrm{mL}$ TRAIL (b), $50 \mathrm{ng} / \mathrm{mL}$ TRAIL (c) or $500 \mathrm{ng} / \mathrm{mLTRAIL}$ (d) are shown. Bars: $100 \mu \mathrm{m}$.
\end{abstract}
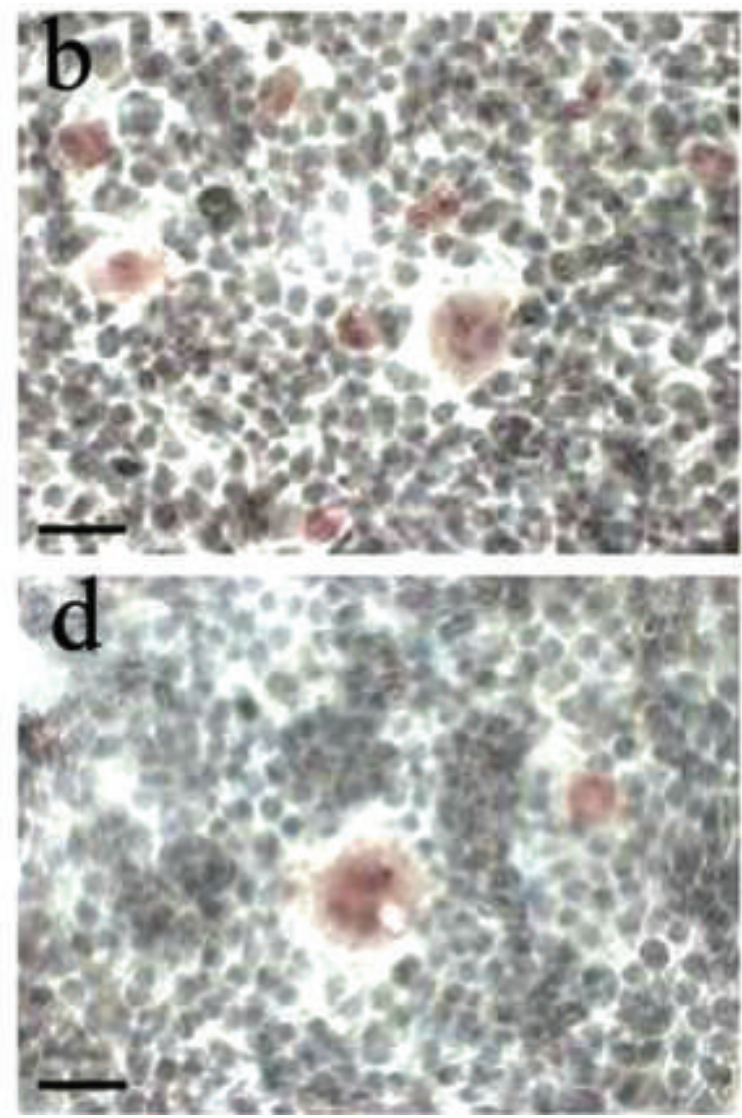

effect of increasing concentrations of recombinant human TRAIL, prepared as described (Zauli et al., 2003), in a previously described model of co-cultures between mouse osteoblasts and RAW264.7 pre-osteoclastic cell line (Nicolin et al., 2006). This culture system shows the advantage that does not require the addition of exogenous cytokines, since it 
spontaneously produces both RANKL and OPG (Nicolin et al., 2006). As shown in Figure 1 A, B, the addition of recombinant human TRAIL had no significant impact on the osteoclast formation up to $50 \mathrm{ng} / \mathrm{mL}$. Only, at high concentrations (500 $\mathrm{ng} / \mathrm{mL})$, recombinant TRAIL significantly $(p<0.05)$ inhibited the formation of osteoclasts. Although we are aware that mouse cells only express the homologue of human TRAIL-R2 while lacking the expression of TRAIL-R1，-R3，-R4 homologues (Zauli and Secchiero, 2006) and therefore experiments in mice cannot be immediately related to the human situation, our current observation, taken together with previous studies (Zauli et al., 2004; Zauli et al., 2008; Roux et al., 2005; Colucci et al., 2004; Colucci et al., 2007; Vitovski et al., 2007) indicate that the relative concentrations and the expression patterns of TRAIL and OPG in the local microenvironment are key determinant in the TRAIL/OPG interactions. Our findings also suggest that only at high but pharmacologically achievable concentrations (Cretney et al., 2007), TRAIL might efficiently inhibit osteoclastogenesis in vivo.

\section{Acknowledgments}

This work was supported by grants from the Programmi di Ricerca Scientifica di Rilevante Interesse Nazionale (PRIN) (F.I.R.B.) (C.C.)

\section{References}

Boyle WJ, Scott SW, Lacey DL. Osteoclast differentiation and activation. Nature 2003;423:337-42.

Colucci S, Brunetti G, Rizzi R, Zonno A, Mori G, Colaianni G, et al. T cells support osteoclastogenesis in an in vitro model derived from human multiple myeloma bone disease: the role of the OPG/TRAIL interaction. Blood 2004;104:3722-30.

Colucci S, Brunetti G, Cantatore FP, Oranger A, Mori G, Pignataro P, et al. The death receptor DR5 is involved in TRAIL-mediated human osteoclast apoptosis. Apoptosis 2007;12:1623-32.

Cretney E, Takeda K, Smyth MJ. Cancer: novel therapeutic strategies that exploit the TNF-related apoptosis-inducing ligand (TRAIL)/TRAIL receptor pathway. Int $\mathrm{J}$ Biochem Cell Biol 2007;39:280-6.

Holen I, Croucher PI, Hamdy FC, Eaton CL. Osteoprotegerin (OPG) is a survival factor for human prostate cancer cells. Cancer Res 2002;62:1619-23.

Nicolin V, Baldini G, Bareggi R, Zweyer M, Zauli G, Vaccarezza M, et al. Morphological features of osteoclasts derived from a co-culture system. J Mol Histol 2006;37:171-7.

Roux S, Lambert-Comeau P, Saint-Pierre C, Lepine M, Sawan B, Parent JL. Death receptors, Fas and TRAIL receptors, are involved in human osteoclast apoptosis. Biochem Biophys Res Commun 2005;333:42-50.

Secchiero P, Gonelli A, Mirandola P, Melloni E, Zamai L, Celeghini C, et al. Tumor necrosis factor-related apoptosis-inducing ligand induces monocytic maturation of leukemic and normal myeloid precursors through a caspase-dependent pathway. Blood 2002; 100:2421-9.

Secchiero P, Melloni E, Heikinheimo M, Mannisto S, Di Pietro R, Icone $A$, et al. TRAIL regulates normal erythroid maturation through an ERK-dependent pathway. Blood $2004 ; 103: 517-22$.

Shipman CM, Croucher PI. Osteoprotegerin is a soluble decoy receptor for tumor necrosis factor-related apoptosis-inducing ligand/Apo2 ligand and can function as a paracrine survival factor for human myeloma cells. Cancer Res 2003;63:912-6.

Vitovski S, Phillips JS, Sayers J, Croucher PI. Investigating the interaction between osteoprotegerin and RANKL or TRAIL: evidence for a pivotal role for osteoprotegerin in regulating two distinct pathways. J Biol Chem 2007;282:31601-9.

Zauli G, Pandolfi A, Monelli A, Di Pietro R, Guarnirei S, Ciabattoni G, et al. Tumor necrosis factor-related apoptosis-inducing ligand (TRAIL) sequentially upregulates nitric oxide and prostanoid production in primary human endothelial cells. Circ Res 2003;9:732-40.

Zauli G, Rimondi E, Nicolin V, Melloni E, Celeghini C, Secchiero P. TNF-related apoptosis inducing ligand (TRAIL) blocks osteoclastic differentiation induced by RANKL+M-CSF. Blood 2004;104:204450.

Zauli G, Secchiero P. The role of the TRAIL/TRAIL-receptors system in hematopoiesis and endothelial cell biology. Cytokine Growth Factor Rev 2006;17:245-57.

Zauli G, Rimondi E, Stea S, Baruffaldi F, Stebel M, Zerbinati C, et al. TRAIL inhibits osteoclastic differentiation by counteracting RANKL-dependent p27Kipl accumulation in pre-osteoclast precursors. J Cell Physiol 2008;214:117-25. 
V. Nicolin et al. 